\title{
Enhancement of contrast sensitivity and losses of chromatic discrimination with tinted lenses
}

\section{Authors:}

Mํㅡㄹ Dolores de Fez (Ph. D.)

$M^{a}$ José Luque*(Ph. D.)

Valentin Viqueira (Ph. D.)

Dpto. Interuniversitario de Óptica, Escuela de Óptica, Universidad de Alicante *Dpto. de Óptica, Facultad de Física, Universidad de Valencia

Number of figures: 7

Date: $2001-11-19$ 


\section{ABSTRACT}

i) Purpose. Tinted lenses for everyday use should not impair visual acuity (VA) and contrast sensitivity (CS) or cause radical changes in colour perception. The main aim of this paper was to compare the performance in contrast detection and colour discrimination tasks of a set of tinted lenses with that of grey filters of equal luminance under D65.

ii) Methods. The contrast sensitivity functions (CSF) of 10 observers were measured using sinusoidal gratings of mean luminance of $13 \mathrm{~cd} / \mathrm{m}^{2}$ by the adjustment method. Colour discrimination thresholds from white $(x=0.313, y=0.330)$, green $(x=0.346$, $y=0.407)$ and blue $(x=0.280, y=0.253)$ were measured along twelve directions in the CIE-1931 xy diagram, with and without lenses.

iii) Results. The results show that the green, brown and blue filters, do not cause significant changes in contrast sensitivity when compared with a grey filter of equal luminance, although chromatic discrimination is disturbed. Yellow and orange filters improve achromatic contrast at certain spatial frequencies, but impair chromatic discrimination.

iv) Conclusions. Compared to grey filters of the same luminance, yellow filters may be useful when enhancement of low achromatic contrasts is desirable, although overall brightness decrements may occur. Nevertheless, these lenses cause tritanlike defects with discrimination losses increasing with the cut-off wavelength. 


\section{INTRODUCTION}

Tinted lenses for everyday use should not cause visual acuity (VA) and contrast sensitivity (CS) losses or radical changes in the perception of colours. These lenses are generally worn with very high illumination levels or with intense scattered light, where a brightness decrement has beneficial effects. Although with all tinted lenses the overall transmittance of the prereceptoral media in the visible spectrum is reduced and the chromatic vision becomes altered to a greater or lesser degree, the visual system has mechanisms that partially compensate these effects. The light adaptation mechanisms may reduce the effect of the transmittance losses in such a way that, at least within a certain range, a reduction of the retinal illumination level does not result in reduced brightness ${ }^{1,2}$ On the other hand, the alterations of colour vision are compensated in a high degree by the colour constancy and illuminant discounting mechanisms ${ }^{1,2}$ and only with certain lenses the resulting chromatic alteration may be important.

The use of tinted lenses is increasing, and besides traditional grey, green and brown tinted glasses, other colours such as blue, violet and yellow are becoming popular. Short wavelength cut-off filters have roused a great deal of interest as they seem to improve vision compared with grey filters ${ }^{3-5}$, although the existence of significant changes has not been conclusively proved. The choice of a particular tinted lens is as likely to be dictated by fashion than by some other subjective criterion of the user. Nevertheless, if a filter is to be worn regularly, it is desirable that the subject's VA, CS and chromatic discrimination should not suffer. For this reason, it is necessary a detailed study of the performance of the more common tinted lenses 
in the market.

Tinted lenses reduce the transmittance of the visible spectrum and change colour perception to a greater or lesser degree $e^{6,7}$. The effect is strongest with yellow and orange filters which cause tritan-like colour vision defects. Cut-off filters have been found to be useful for some low vision subjects ${ }^{8-10}$, probably due to their glare reduction effects. The use of these filters by healthy subjects has been justified by an alleged increase of image contrast. This improvement may be attributed to a decrement of the chromatic aberration effects ${ }^{11-15}$, to a brightness increment ${ }^{1,7,16-20}$, to scattering reduction ${ }^{11,12,21}$, or to a decrement of lenticular fluorescence ${ }^{22-24}$.

The effects of chromatic aberration are more noticeable in a high spatial resolution task. Since the VA, one of the tasks most sensitive to the presence of this aberration, is not improved with the use of tinted lenses, the effect of the reduction of chromatic aberration due to the lens probably will not improve the subject's performance in contrast discrimination tasks ${ }^{11-15}$. Nevertheless, it is likely that macula lutea and eye-lens fluctuations are enough to compensate reasonably the chromatic aberration of the eye $\mathrm{e}^{13}$. Even though most tinted filters significantly reduce stimulus luminance, brightness increments have been found, and probably such increments play a role in the feeling of improvement in vision quality reported by some subjects $^{1,7,16-20}$. According to Kinney ${ }^{17}$, the origin of this enhancement is the response increment of the opponent chromatic mechanisms due to the removal of the negative contribution of short-wavelengths. It has also been observed that pupil size increases with these filters, which may also contribute to the brightness increment ${ }^{18}$. The decrement of blue radiation arriving to the eye reduces also the scattering effects, 
which would justify the good performance of these filters under glare and bad atmospheric conditions ${ }^{11,12}$. Nevertheless, the contrast enhancement due to scattering decrement has not till now been shown experimentally, although some authors suggest a slight improvement of contrast sensitivity to low spatial frequencies ${ }^{21}$. This property would justify the predilection of skiers and professional drivers for these lenses in foggy weather, because under these conditions detail perception is less important that the capability of detecting the presence of shapes and volumes, that is, low spatial frequencies. Finally, it has been found that the shortwavelengths removed by the cut-off filters produce fluorescence effects in the chromophore-containing proteins present in the eye lens ${ }^{22-24}$.

It is reasonable to believe that the different contributions of the phenomena discussed above result in a contrast increment. Paradoxically, however, and in spite of numerous works on the subject, it has not been possible to prove experimentally a significant improvement either in the CSF or in the VA, except for very particular setups, such as yellow tests on blue backgrounds ${ }^{25}$. Cut-off filters do seem to increase low contrasts when compared with grey filters $(20 \%$ increments for 0.1 contrast whereas no differences are found for contrasts greater than 0.6$)^{4}$. On the other hand, slight contrast sensitivity increments have been found in mesopic and, more perceptibly, photopic levels ${ }^{5}$.

As can be seen, the works in this field are numerous. However, most of the papers compare a small number of filters or are even limited to a single cut-off filter and are often concerned with a single aspect of visual perception, such as contrast sensitivity or brightness. The main aim of this paper is to compare the performance of 
a variety of filters in contrast detection and colour discrimination tasks. Each filter has been compared with a reference grey filter of the same luminance under D65, to determine if the changes observed in the CSF and in the colour discrimination ellipses are due to luminance or to colour effects.

\section{METHODS}

Stimuli were generated on a 20"-Mitsubishi HL7955 CRT screen, controlled by a VSG 2/3F graphic card from Cambridge Research Systems (CRS). The screen was calibrated using a photometer (CRS) controlled by specific software that automatically calibrates and gamma-corrects the monitor. The brightness and contrast settings of the monitor were fixed and the controls sealed to avoid accidental changes.

Seven tinted lenses commercially available were used in the experiments: three grey filters with different densities (GREYA, GREYB and GREYC) and four chromatic filters (BLUE, GREEN, BROWN and YELLOW). Two lenses used by lowvision patients were also included (CPF450 and CPF527). The spectral transmittance of each (Figure 1) was measured with an ATI Unicam UV2/200 spectrocolorimeter, from 300 to $850 \mathrm{~nm}$, in $2 \mathrm{~nm}$ steps. In the calculations performed in this work only the visible range, from 350 to $825 \mathrm{~nm}$ was considered. The YELLOW, CPF450 and CPF527 are short cut-off wavelength filters, with very low transmittance $(<10-15 \%)$ below 420, 450 and $527 \mathrm{~nm}$, respectively (Figure 1a). The BLUE filter has transmittance maxima at 450 and $780 \mathrm{~nm}$. The BROWN and GREEN filters are highpass, but their transmittances in the $400-600 \mathrm{~nm}$ range are not negligible (Figure 1b). 
Figure 1

The CSF was measured using "PsychoWin 2.25" software from CRS, which generated sinusoidal gratings (average luminance $13 \mathrm{~cd} / \mathrm{m}^{2}$ ) at spatial frequencies of $1,2,3,4,6,8.8,12.6$ and $19 \mathrm{cpd}$, presented to the observer in random order. The frequency range used covered the range of a normal observer and included several frequencies in the maximum sensitivity region. A circular mask subtending two degrees limited the stimuli. The observer, placed at three metres from the screen, determined by the adjustment method the minimum contrast below which the test appeared uniform, without any time limitation. For each frequency, the grating contrast was initially unity, but after each measurement the initial contrast value of the test was reduced according to the threshold value determined in the previous measurement. The final contrast threshold was determined as the mean of four measurements.

Colour vision of the observers was assessed with and without the tinted lenses using CRS's Colour Vision Test ${ }^{26-28}$. The discrimination threshold from white $(x=0.313, y=0.330)$, green $(x=0.346, y=0.407)$ and blue $(x=0.280, y=0.253)$ was measured along twelve directions in the CIE-1931 xy diagram. Each stimulus was formed by circular spots of random size and luminance shaping a letter $\mathrm{C}$, whose gap subtended one degree, differing in chromaticity from a background of any of the three aforesaid colours. The random variation of luminance, in the $8-17 \mathrm{~cd} / \mathrm{m}^{2}$ range and with a mean value of $13 \mathrm{~cd} / \mathrm{m}^{2}$, ensured that the test was detected only if a colour difference between test and background was perceived. The size of the spots and 
the luminance distribution were kept constant for each background, whereas the chromaticity of the letter $\mathrm{C}$ was made to differ from that of the background along 12 directions on the chromaticity diagram. The task of the observer was to report the position of the gap in the $\mathrm{C}$, which changed randomly. The stimulus disappeared after three seconds; the program treated a lack of response within this interval as a failure to detect the test. A staircase procedure was used to determine the minimum colour difference that made the observer's response reliable for a given colour direction. Once the discrimination thresholds from each given background were measured, the Colour Vision Test determined the parameters of the ellipse that fits best the data.

\section{Experimental sessions}

Three male and seven female observers, with ages ranging from 22 to 34 took part in the experiment. Both the Farnswoth-Munsell 100 Hue test and CRS's Colour Vision Test determined that the colour vision of all the observers was normal. Two of the observers are the authors of this paper and the rest were experienced observers but naïve regarding the aim of the experiment.

In each session, the observer was preadapted to a $13 \mathrm{~cd} / \mathrm{m}^{2}$ achromatic homogeneous field $(x=0.29 y=0.31)$ subtending $30^{\circ}$ generated by an ordinary $14^{\prime \prime}-$ CRT monitor in a dark room. The observer viewed the adaptation stimulus for three minutes, wearing the same lenses that would use during the experiment, after which was placed at $3 \mathrm{~m}$ from the stimulus generator for the measurement session.

The measurement of each CSF took about 10 minutes. The measurement of 
the chromatic thresholds around a given background varied from 15 to 20 minutes. The measurement sessions were tailored so that they never took longer than $25-30$ minutes.

\section{RESULTS}

\section{CSFs}

The average contrast sensitivity of the observers without lenses was better than with any of the lenses used in the experiment, except if the spatial frequency is high, when the contrast sensitivity with the yellow lenses was largest (fig. 2). The greatest reduction in overall contrast was with the BROWN and GREEN filters.

Figure 2

The influence of the tinted lenses on the CSF may result from the contributions of a change in the retinal illumination and of the selective spectral filtering of light. To separate these two effects, in what follows, we will compare each tinted lens with a grey filter of the same luminance L, computed as:

$$
L=683 \int \tau(\lambda) s(\lambda) \mathrm{V}(\lambda) \mathrm{d} \lambda
$$

where $\tau(\lambda)$ is the filter transmittance, $s(\lambda)$ the spectral radiance of illuminant D65 and $\mathrm{V}(\lambda)$ the CIE photopic standard observer. With this criterion, the reference grey filters are GREYA for the CPF450 $\left(\mathrm{L}_{\text {ga }} / \mathrm{L}_{450}=1.15\right)$ and $\mathrm{YELLOW}\left(\mathrm{L}_{\text {ga }} / \mathrm{L}_{\text {yellow}}=1.01\right)$ lenses, GREYB for CPF527 $\left(L_{g b} / L_{527}=1.10\right)$ and GREYC for the BLUE $\left(L_{g c} / L_{b l u e}=0.81\right)$, 
BROWN $\left(\mathrm{L}_{g c} \mathrm{~L}_{\text {brown }}=0.97\right)$ and GREEN $\left(\mathrm{L}_{g c} / \mathrm{L}_{\text {green }}=1.11\right)$ lenses. The luminance of the BLUE filter, nevertheless, is slightly larger than that of GREYC, and this must be kept in mind in the discussion of the results. Nevertheless, retinal illumination changes in the range considered will probably have little effect on the CSF, as can be seen from our results with the reference grey filters (Figure 3). Although with the GREYA filter the sensitivity is greatest, the differences between GREYC and GREYB do not seem consistent with a retinal illumination change because the two curves are very close to each other and cross over occasionally.

Figure 3

In Figure 4a-c we represent the ratios between the CSFs obtained with the coloured filters and with the corresponding reference. In Figure 4a, we represent these ratios for the YELLOW and CPF450 filters. The sensitivity changes that occur for 3, 4, 6 and $8.8 \mathrm{cpd}$ are small. When spatial frequency is very low (1 and $2 \mathrm{cpd}$ ), the contrast sensitivity with these tinted lenses, and particularly with the YELLOW filter, is notably worse than with the GREYA filter. For 4 and $12.6 \mathrm{cpd}$ the sensitivity with the CPF450 filter is better than with the grey filter, and the same happens, although in a lesser degree, with the YELLOW filter. In fact, for $4 \mathrm{cpd}$ the relative improvement induced by the YELLOW filter is negligible. To determine whether a ratio different from one was significant, we performed an one-way ANOVA with the results of our ten observers with the tinted filters and the reference grey lens for each frequency. Only the differences between CPF450 and GREYA were statistically significant $(p<0.05)$, and this just for the higher frequencies $(8.8,12.6$ and $19 \mathrm{cpd})$. We may conclude that the CPF450 filter significantly improves sensitivity at $12.6 \mathrm{cpd}$. 
The orange CPF527 filter is compared with GREYB in Figure 4b. The behaviour is similar to that observed with CPF450, the frequency for which the sensitivity increment is largest changing from 12.6 to $8.8 \mathrm{cpd}$. Nevertheless, the differences between CPF527 and GREYB are not statistically significant ( $p>0.05)$.

Finally, the three remaining filters, GREEN, BLUE and BROWN are compared with GREYC in Figure 4c. These three filters behave similarly, all of them presenting a maximum loss of sensitivity at $6 \mathrm{cpd}$. The three curves have approximately the same shape and only differ in a scaling factor, with the CSF obtained with the BROWN filter at the bottom and with the BLUE at the top. The sensitivity losses at high frequencies become smaller, so that at $19 \mathrm{cpd}$ with the GREEN filter and above $8.8 \mathrm{cpd}$ with the BLUE filter, the sensitivity is greater than with the GREYC filter. At low spatial frequencies, the sensitivity obtained with the filters is always smaller than or equal to the sensitivity measured with the grey filter. The differences in behaviour between these four filters are statistically significant at $3 \mathrm{cpg}$ only $(p<0.05)$.

Figure $4 a-c$

\section{Discrimination ellipses}

The discrimination ellipses measured with each filter are plotted in Figure 5a-c. Again, filters have been classed according to their luminance.

Figure 5a-c 
Chromatic discrimination was always best without lenses and better with the grey than with the coloured filters. The sizes of the discrimination ellipses obtained with the grey filters and without lenses are similar. An one-way ANOVA was performed to test for statistically significant differences between the values of the parameters of the discrimination ellipses (mayor and minor axis and orientation) of our ten observers obtained with the tinted lenses and the corresponding reference grey filter. In all cases, the differences in the major axis of the ellipses are statistically significant $(p<0.05)$. The results about the orientation of the ellipse are less conclusive because with the grey filters the discrimination ellipses are practically circumferences and therefore the orientation is not well defined; this is apparent in the large dispersion of this parameter both for each observer and for the whole sample considered.

The major axis of the ellipses around the white and the green backgrounds obtained with the BLUE filter lies on a red-green direction, although it does not coincide either with a protan or a deutan confusion line. With this lens and the blue background, the major axis of the ellipse lies near the corresponding tritan confusion line, although it does not coincide with it. With the rest of the coloured filters, the major axis of the ellipse is almost vertical, forming an angle of approximately $20^{\circ}$ with the tritan confusion line. The major axis of the ellipses obtained with CPF527 lies closest to the tritan confusion lines.

To separate the effects due to the colour of the filters from those due to their luminance, the discrimination ellipses of the coloured filters were compared with those of a grey filter of a similar luminance. The minor axes of the ellipses obtained 
with the coloured filters and the reference grey filter were similar. In the region of worst discrimination, the discrimination losses associated to the use of the CPF filters were approximately twice that of the other coloured filters. In all cases, discrimination was worst with CPF527.

\section{DISCUSION}

Tinted lenses should not significantly impair the performance of the visual system. This study examined contrast sensitivity and chromatic discrimination. Once the effect of our six coloured filters on these two parameters is known, we shall discuss some aspects of spatial vision. In particular, we wanted to ascertain how tinted lenses would affect the perception of achromatic complex objects. To this end, we have filtered a set of four different black-and-white photographs with the mean CSF obtained with each filter and simulated the appearance of the resulting image (in Figures 6 and 7only two illustrative examples are shown). The effect of each filter in chromatic discrimination will serve finally to determine which of the tinted lenses used combines an enhancement of achromatic contrast sensitivity with the smallest impairment of chromatic vision.

We have assumed that the visual system (VS) may be described in the spatial domain by a two-dimensional version of the $\operatorname{CSF}^{29}, \operatorname{CSF}_{\mathrm{vs}}\left(v_{x}, v_{y}\right)$. If $L_{0}(x, y)$ is the original spatial luminance distribution in the scene and $L_{v S+l e n s}(x, y)$ is the image perceived by a subject wearing tinted lenses, then: 


$$
\operatorname{TF}\left[L_{\text {VS+lens }}(x, y)\right]=\operatorname{CSF}_{V_{S+l e n s}}\left(v_{x}, v_{y}\right) \operatorname{TF}\left[L_{0}(x, y)\right]
$$

where $\operatorname{CSF}_{\mathrm{VS}+\text { lens }}\left(v_{\mathrm{x}}, v_{\mathrm{y}}\right)$ is the CSF of the subject with the lens.

The perceived image $\operatorname{LvS}_{v+e n s}(x, y)$ will be simulated by the spatial luminance distribution $L_{s}(x, y)$, verifying that the subject without lenses will perceive the same viewing $L_{s}(x, y)$ as when viewing $L_{0}(x, y)$ with the lenses. Therefore, $L_{s}(x, y)$ must verify:

$$
\operatorname{TF}\left[L_{v S+l e n s}(x, y)\right]=\operatorname{CSF}_{v S}\left(v_{x}, v_{y}\right) \operatorname{TF}\left[L_{s}(x, y)\right]
$$

From (2) and (3)

$$
\mathrm{L}_{\mathrm{S}}(\mathrm{x}, \mathrm{y})=\mathrm{TF}^{-1}\left[\operatorname{CSF}_{\mathrm{VS}+\text { lens }}\left(v_{\mathrm{x}}, v_{\mathrm{y}}\right) \operatorname{TF}\left[\mathrm{L}_{0}(\mathrm{x}, \mathrm{y})\right] / \operatorname{CSF}_{\mathrm{VS}}\left(v_{\mathrm{x}}, v_{\mathrm{y}}\right)\right]
$$

Note that this procedure does not completely remove the influence of the reader's CSF, which might significantly differ from the $\mathrm{CSF}_{\mathrm{Vs}}$ obtained as the mean of our 10 observers.

In Figure 6 we show the results of the simulation with the nine filters used. Compared with GREYA, filters CPF450 and YELLOW enhance the borders of the image (consider, for instance, the sharpness of the folds in the hatband and of the features of the model in the three images). The same effect is visible when comparing GREYB with the orange CPF527 filter, although, on the other hand, the dark background becomes more uniform, the shadow of the left-hand rafter merging with the wall. The differences between the images obtained with the darker filters (GREYC, GREEN, BROWN and BLUE) are small and seem strictly confined to 
image brightness, which is slightly higher with the BLUE filter. From this image, we might conclude that the CPF450, YELLOW and CPF527 filters enhance the contrast of the scene. Nevertheless, this kind of assertion must be treated with caution, since the effect of the filter depends on the frequency content of the scene. For instance, in Figure 7, the image on the left, obtained with GREYA, shows more clearly the barrel of the key in the middle than the other two filters, with which this key would appear to be thinner.

\section{CONCLUSIONS}

In conclusion, the lenses whose luminance is comparable to the GREYC filters do not improve contrast and decrease slightly image brightness; with lighter filters, of luminance comparable to GREYB or GREYA, the coloured yellow and orange filters are capable of enhancing low contrasts, although brightness may decrease. Thus, the CPF450, CPF527 and YELLOW filters may be useful when enhancement of low achromatic contrasts for middle and high spatial frequencies is desirable. Nevertheless, we have shown that these lenses cause a tritan-like defect in the wearer, with discrimination losses in the yellow-purplish region that are largest for the CPF527 and smallest for the YELLOW filter. These results are consistent with the literature ${ }^{3-7,16-20}$. The use of the YELLOW filter would be more advisable, as it is capable of enhancing contrast with minimum disturbance of the user's chromatic vision.

The GREEN, BROWN and BLUE filters do not introduce significant brightness and contrast changes when compared with their reference grey filter. All three filters 
cause chromatic discrimination losses, but these are of the same order as with the reference filter. The changes in colour vision induced by these filters cannot be classified either as tritan, deutan or protan, because the major axis of the discrimination ellipses are not oriented along any particular dichromatic confusion line. The chromatic discrimination losses along these axis are slightly greater than with the reference grey filter. In this case, the use of the grey filter would be more advisable than any of these three tinted lenses. 


\section{REFERENCES}

1. Fairchild MD. Color appearance models, Addison Wesley Longman Inc. Massachusetts 1998.

2. Hunt RWG. The reproduction of colour, Fountain Press, England 1995.

3. Kelly SA. Effect of yellow-tinted lenses on brightness, J Opt Soc Am 1990;7:19051911.

4. Rabin J, Wiley R. Differences in apparent contrast in yellow and white light, Ophthal Physiol Opt 1996;16:56-72.

5. Yap M. The effect of a yellow filter on contrast sensitivity, Ophthal Physiol Opt $1984 ; 4: 227-232$.

6. Aarnisalo E. Effects of yellow filters glasses on colour discrimination of normal observers and on the illumination level, Acta Ophthalmol 1987;65:274-278.

7. Wolffsohn JS, Cochrane AL, Khoo H, Yoshimitsu Y, Wu S. Contrast is enhanced by yellow lenses because of selective reduction of short-wavelength light, Opt Vis Sci 2000;77:73-81.

8. Provines WF, Harville B, Block M. Effects of yellow optical filters on contrast sensitivity function of albino patients, J Am Optom Assoc 1997;68:353-359. 
9. Leguire LE, Suth S. Effect of light filters on contrast sensitivity function in normal and retinal degeneration subjects. Ophthal Physiol Opt 1993;13:124-128.

10. Zigman, S. Light filters to improve vision. Opt Vis Sci 1992; 69:325-328.

11. Walls GL, The vertebrate eye and its adaptative radiation, 1942, Bloomfield Hills, Michigan, Cranbrook Institute of Science.

12. Walls GL, Judd HD, The intraocular colour-filters of vertebrates, $\mathrm{Br} \mathrm{J}$ Ophthalmol 1933;17:641-675, 17:705-725.

13. Sivak JG, Bobier WR. Effect of a yellow ocular filter on chromatic aberration: the fish eye as an example, Am J Optom Physiol Optics 1978;55:813-817.

14. Reading VM, Weale RA. Macular pigment and chromatic aberration, J Optom Soc Am 1974;62:231-234.

15. Pokorny J, Graham CH, Lanson RN. Effect of wavelength on foveal grating acuity, J Opt Soc Am 1968;58:1410-1414.

16. Kinney JAS, Schlichting CL, Neri DF, Kindness SW. Various measures of the effectiveness of yellow goggles, Tech Rep NSMRL-941, October 1980, Naval Submarine Medical Research Laboratory, Groton, CT, USA 
17. Kinney JAS, Schlichting CL, Neri DF, Kindness SW. Reaction time to spatial frequencies using yellow and luminance-matched neutral gogles, Am J Optom Vis Sci 1983;60:132-138.

18. Chung S, Pease PL. Effect of yellow lenses on pupil size, Optom Vision Sci (suppl) 1993;70:136.

19. Provines WF, Rahe AJ, Block MG, Pena T, Tredici TJ. Yellow lens effects upon visual acquisition perfomance, Aviat Space Env Med 1992;63:561-564.

20. Rieger MD. Improvement of contrast sensitivity with yellow filter glasses, Can $\mathrm{J}$ Ophthalmol 1992;27:137-138.

21. Ding C, Sun B, Zheng Y. Contrast Sensitivity of several blindness-inducing eye disease and the influence of tinted filter lens. Abstract of Ghung Hua Yen Ko Tsa Chih 1997;33:286-288.

22. Miller D. The effect of sunglasses on the visual mechanism, Surv Ophthalmol 1974;19:38-44.

23. Lermann S. Lens proteins and fluorescence, Isr J Med Sci 1973; 8: 8-9.

24. Lermann S. Radiant Energy and the Eye, MacMillan, New York, 1980;1:142-143.

25. Luria SM. Vision with chromatic filters, Am. J. of Optom. and Arch. of Am. Acad. 
of Optom., 1972;October:818-829.

26. Mollon JD, Reffin JP. A computer-controlled colour vision test that combines the principles of Chibret and Stilling, J Physiol 1989; 414, 5P.

27. Reffin JP, Astell S, Mollon JD. Trials of a computer-controlled colour vision test that preserves the advantages of pseudoisochromatics plates. In Colour Vision Deficiences X, 69-76. 1991 Kluwer Academic Publishers, Dordrecht.

28. Regan BC, Reffin JP, Mollon JD. Luminance noise and the rapid determination of discrimination ellipses in colour deficiency. Vis Res 1994;10:1279-1299.

29. Malo J, Felipe A, Luque MJ, Artigas JM. On the intrinsic two-dimensionallity of the CSF and its measurement. J Optics 1994;25:93-103.

MD de Fez

Mailing address: Departamento de Optica, Universidad de Alicante, Campus de San Vicente, Ap. 99, 03080, Alicante (Spain)

Email address: dolores.fez@ua.es 


\section{FIGURE LEGENDS}

Figure 1: Transmittances of the tinted lenses used in our study: a) CPF $450(-)$, CPF

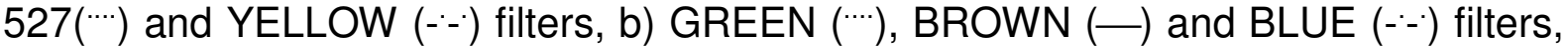
c) GREYA (--), GREYB (') and GREYC (-) filters.

Figure 2: Mean value and standard deviation of the CSFs of 10 observers for each of the tinted lenses, compared with the mean CSF measured without filters.

Figure 3: CSF (mean $\pm s d$ ) of the average observer measured with the three grey filters, compared with the mean CSF measured without filters.

Figure 4: Contrast sensitivities of each coloured filter relative to the corresponding

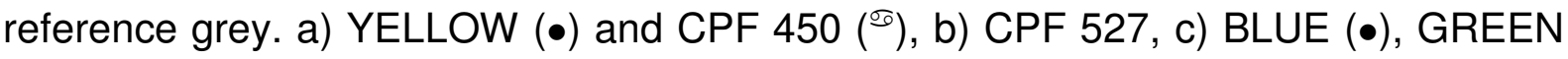

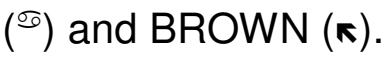

Figure 5: Average discrimination ellipses obtained with the different tinted lenses and the corresponding reference grey filter: a) GREYA (O), YELLOW (O) and CPF 450 (०); b) GREYB (O) and CPF 527 (O); c) GREYC (O) and GREEN (०), BROWN (O) and BLUE (0). Backgrounds are labelled 1 (white), 2 (green) and 3 (blue).

Figures 6: Simulation of the appearance of a black-and-white scene when seen by our average observer through the different tinted lenses 1) image seen without lenses 2) GREYA, 3) CPF 450, 4) YELLOW, 5) GREYB, 6) CPF 527, 7) GREYC, 8) GREEN, 9) BROWN, 10) BLUE. 
Figure 7: Simulation of the appearance of a black-and-white scene when seen by our average observer through different filters of similar luminance. From left to right, GREYA, CPF 450 and YELLOW. The information about the thickness of the barrel of the middle key is lost with the two yellow filters. 
a)

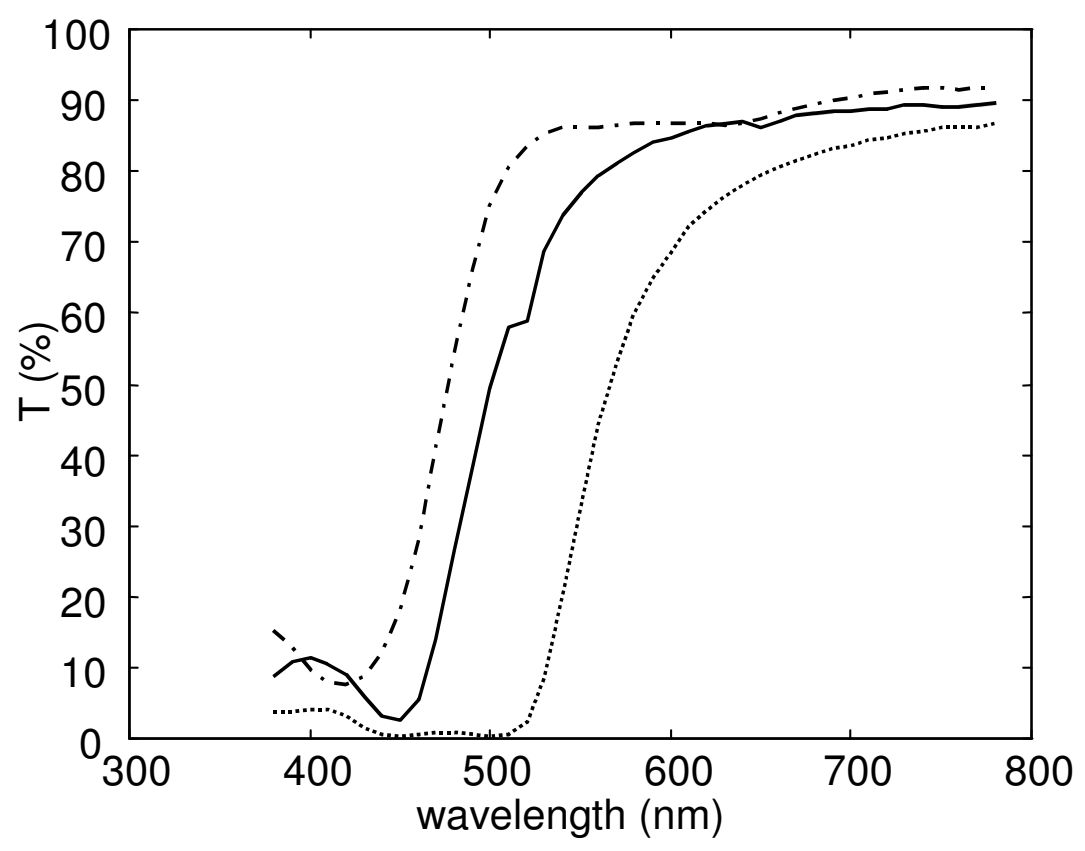

b)

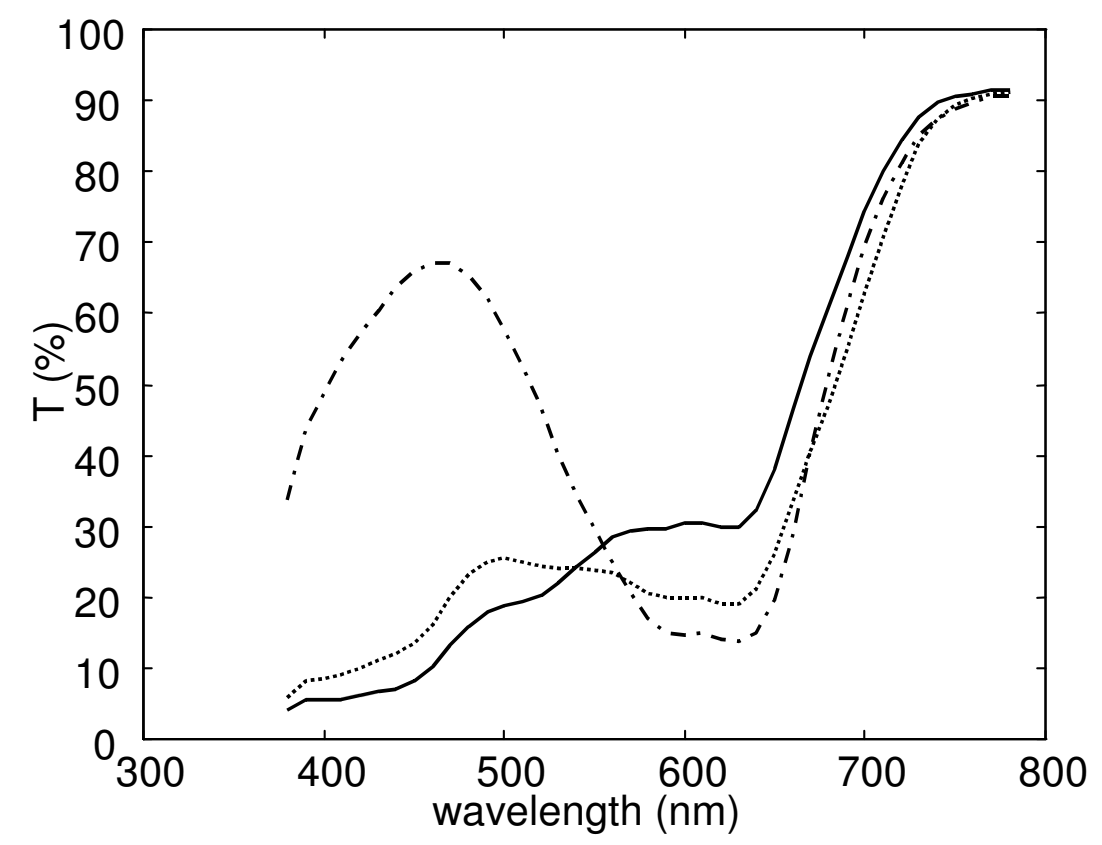


c)

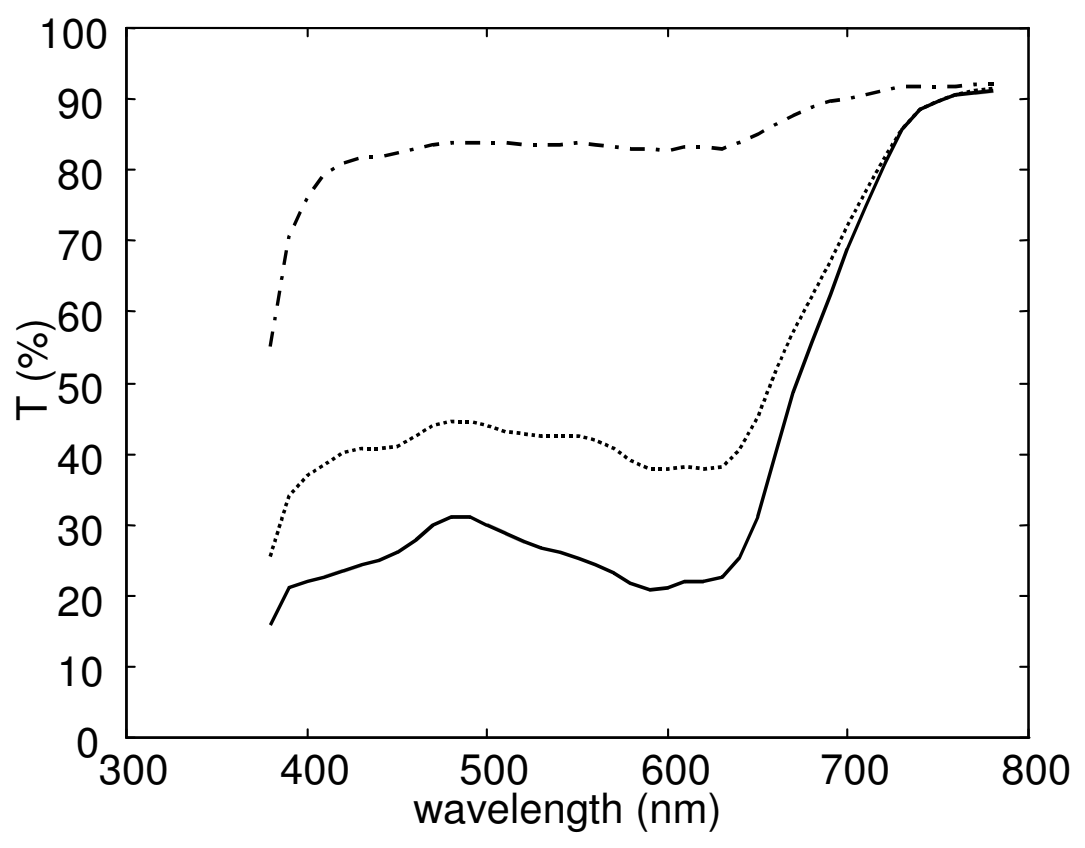




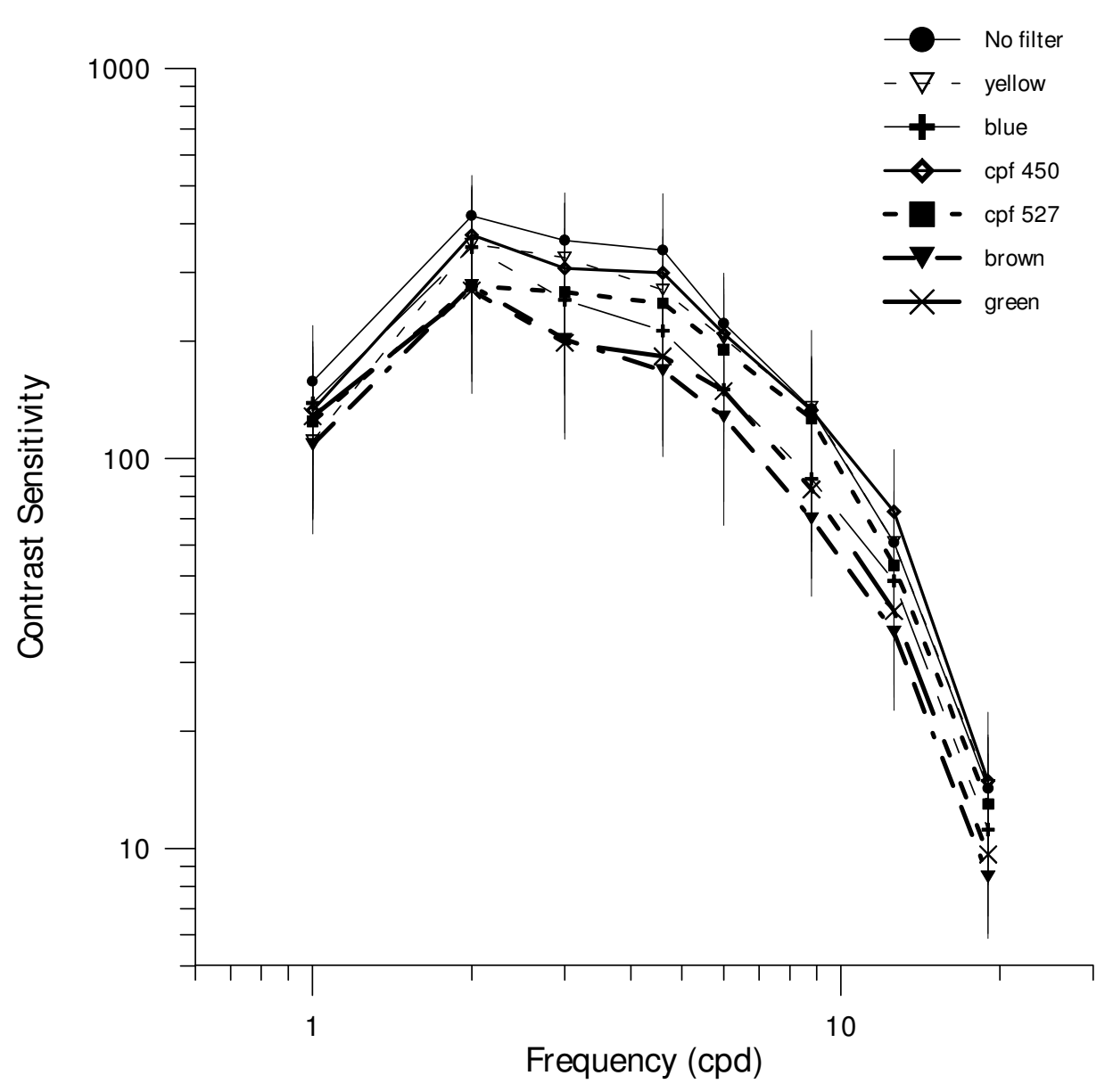




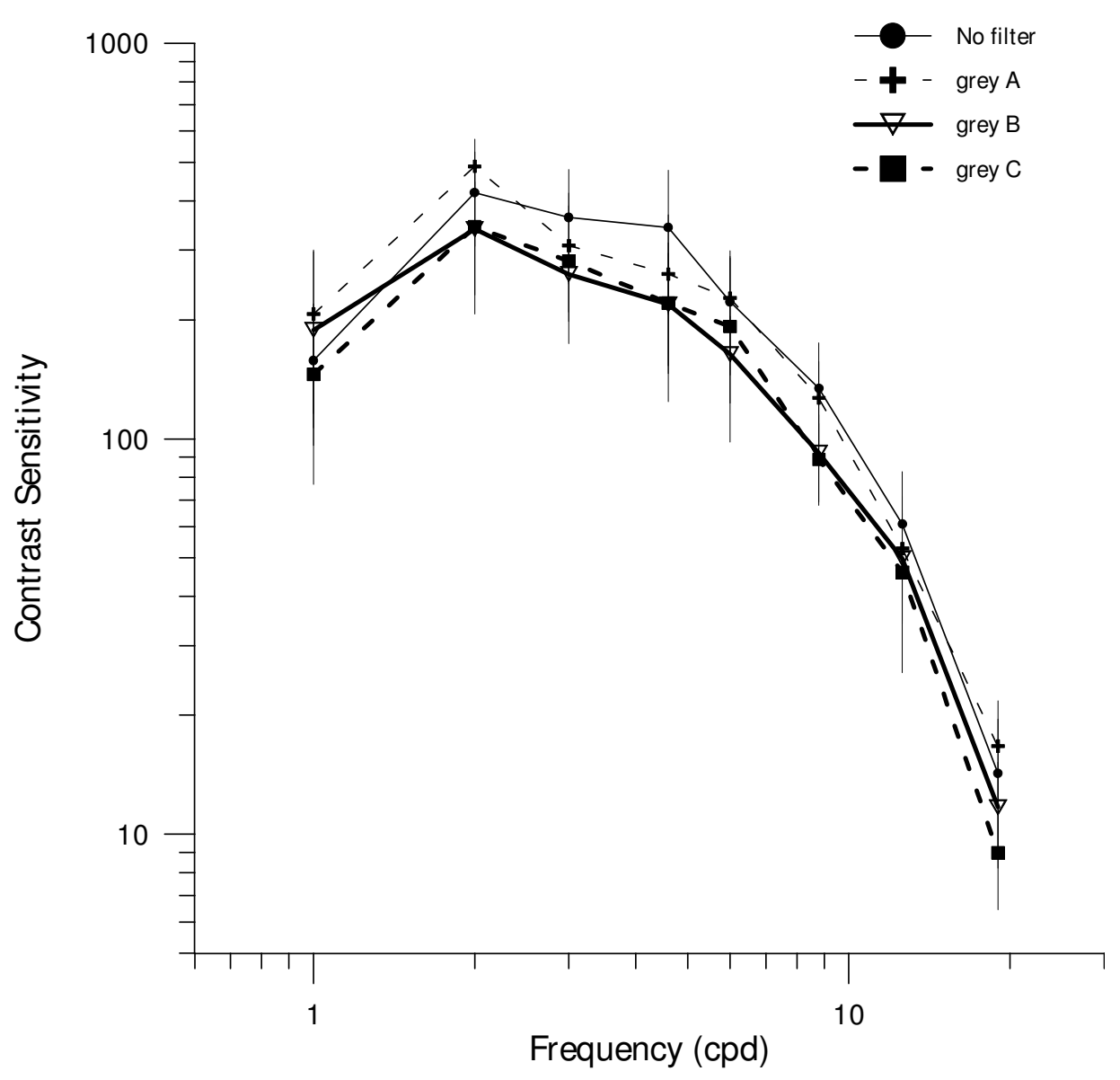


a)

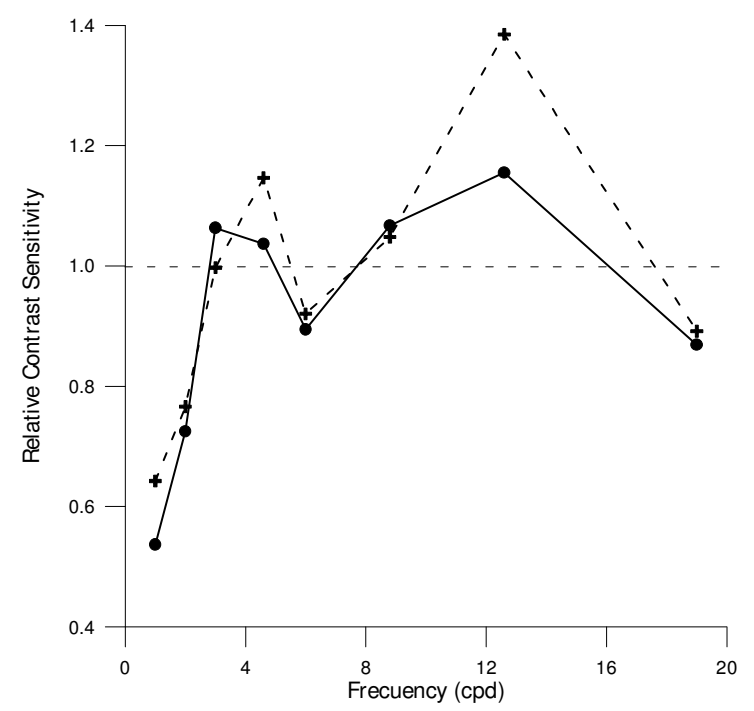

b)

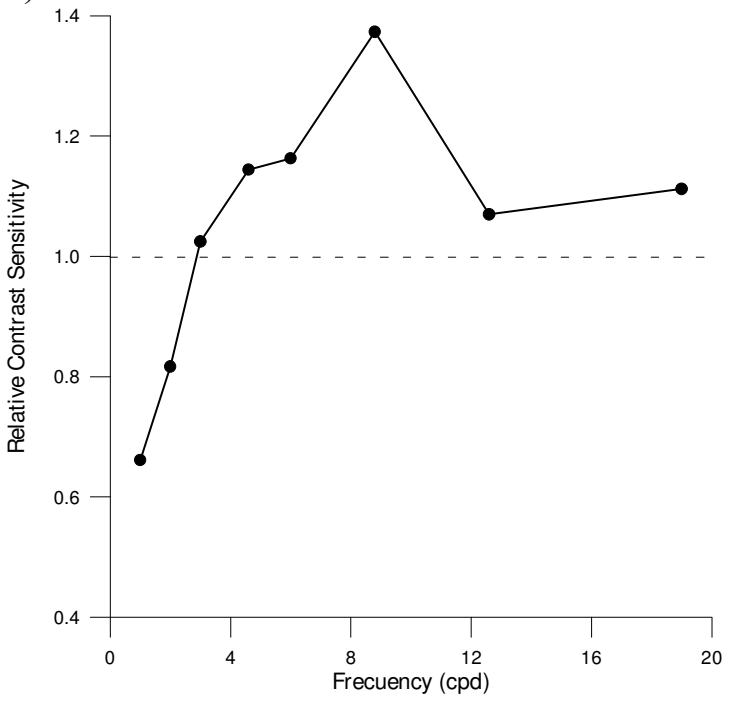




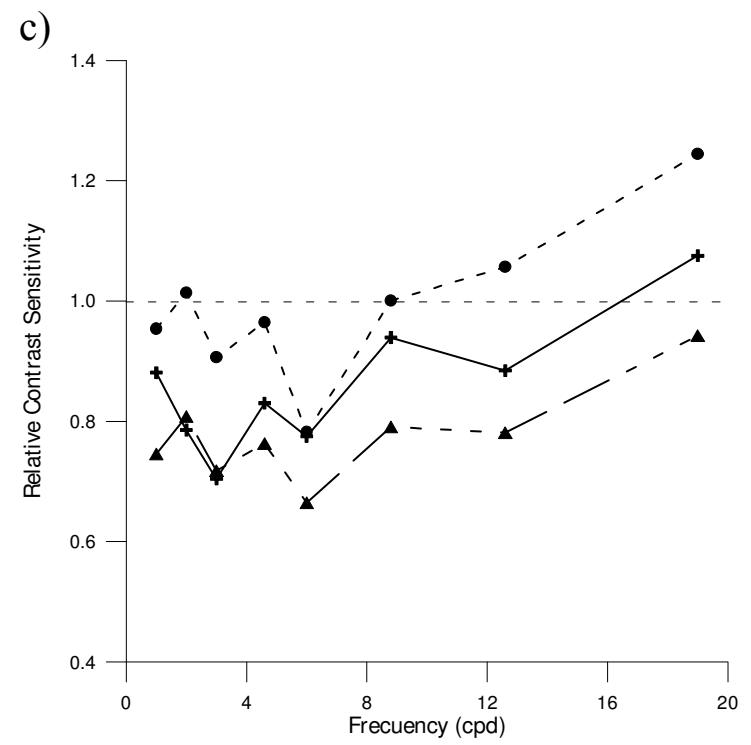


a)

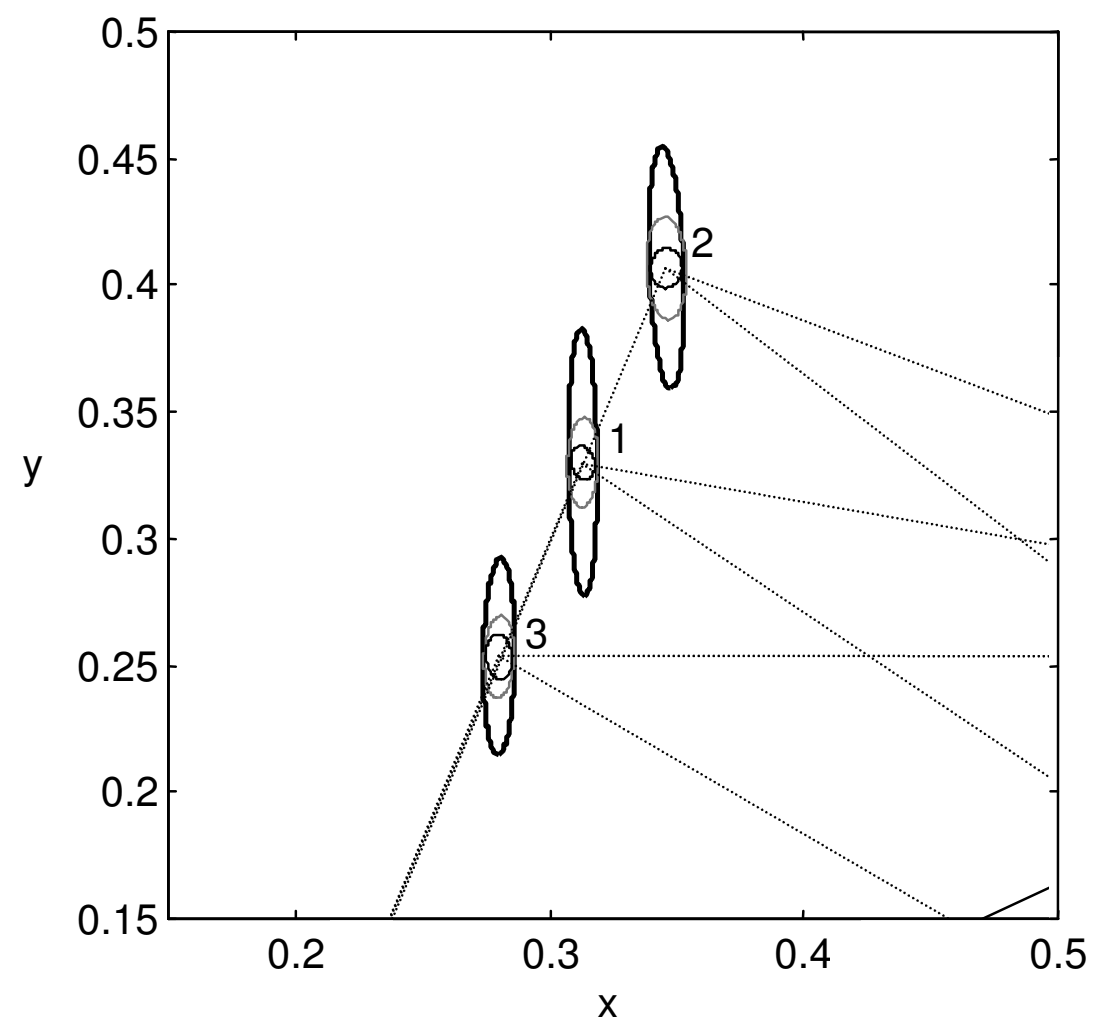

b)

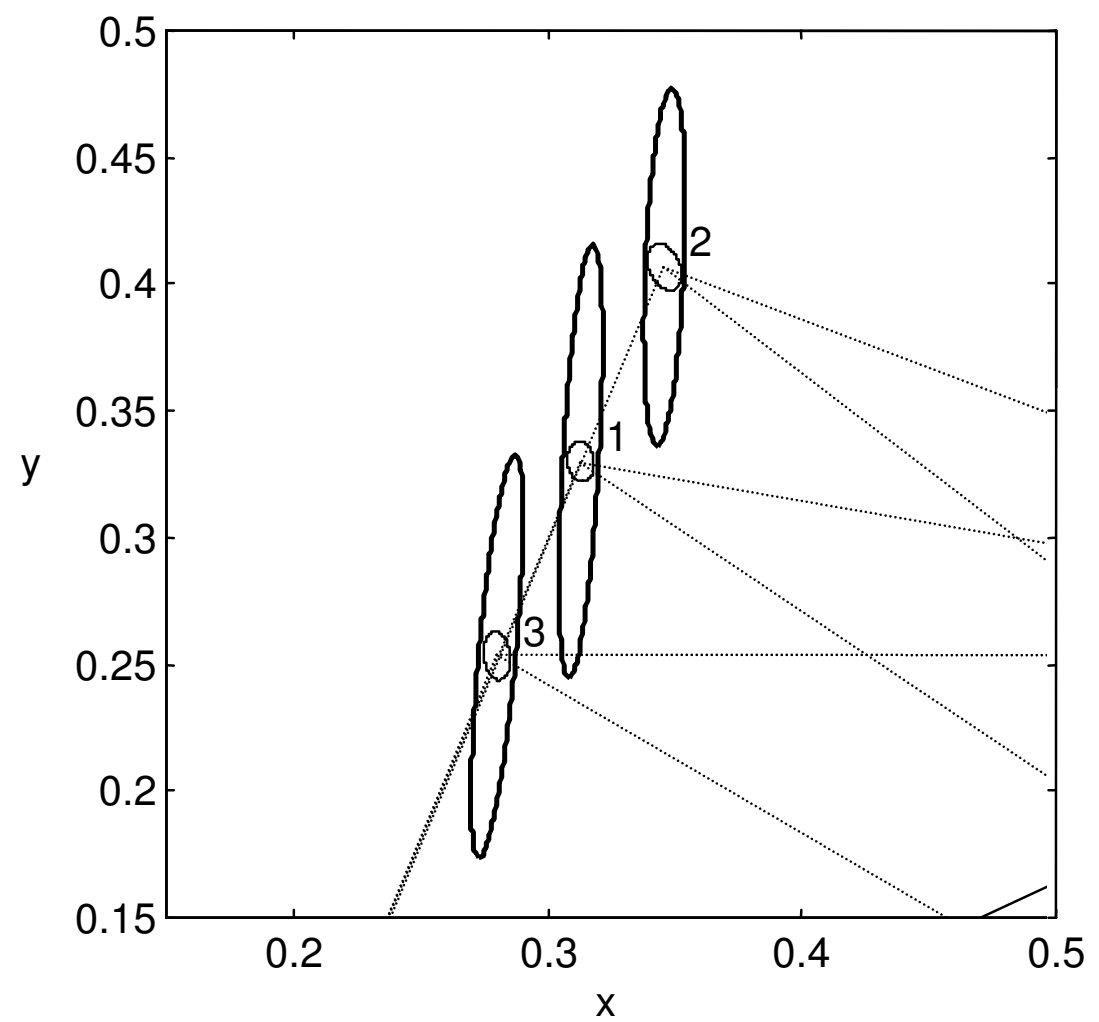


c)

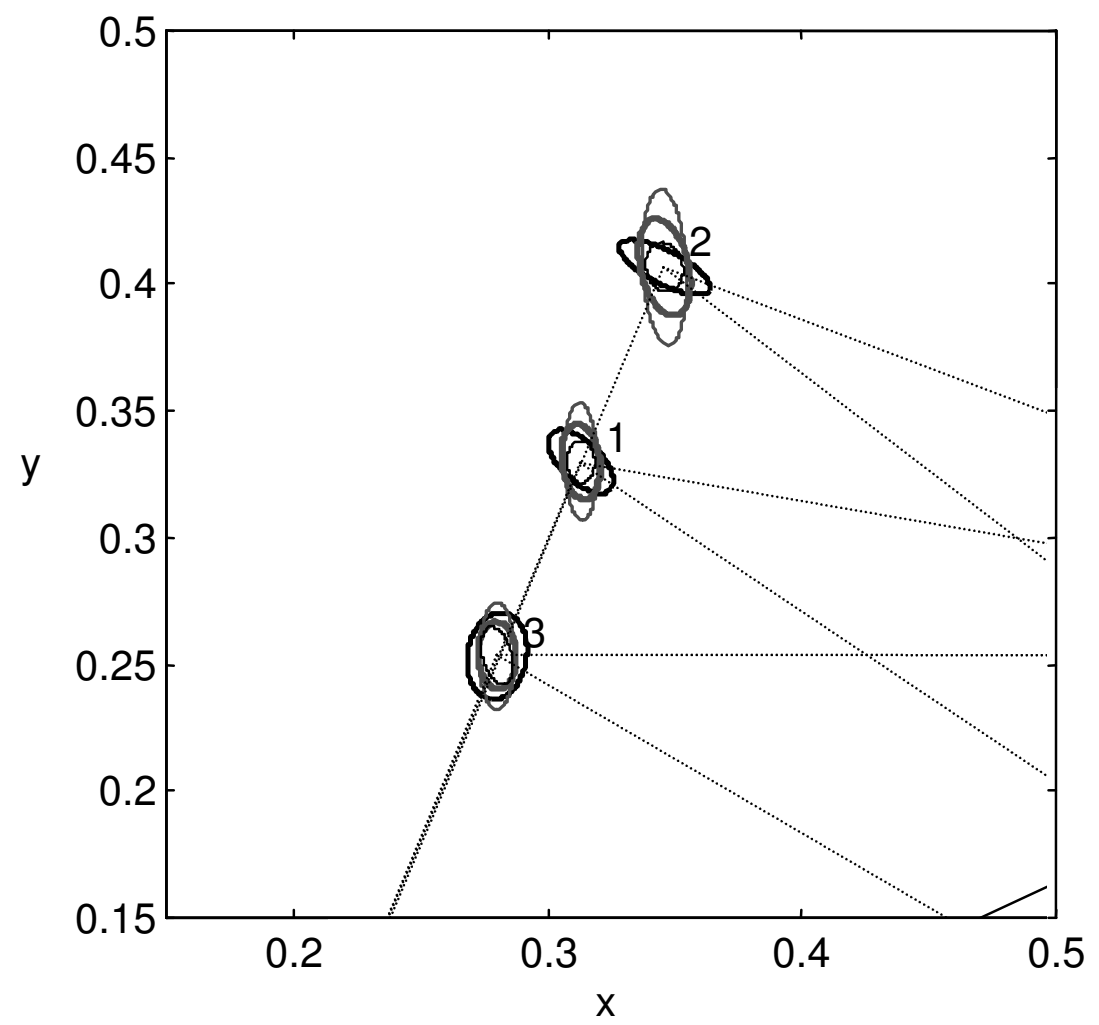


1)

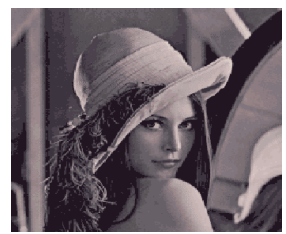

2)

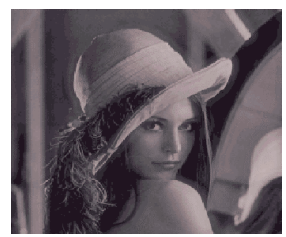

3)
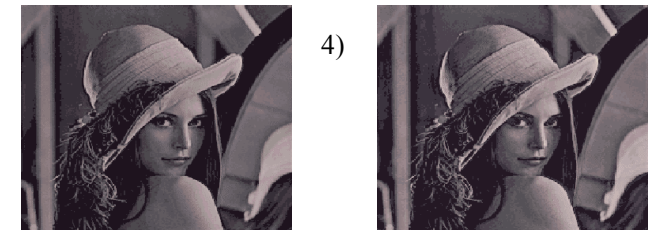

5)

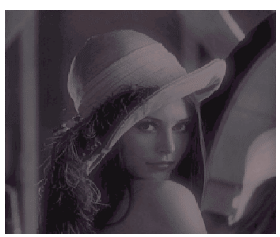

6)

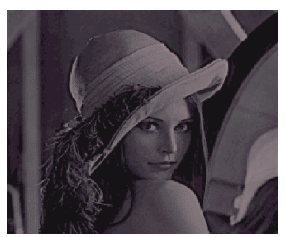

7)

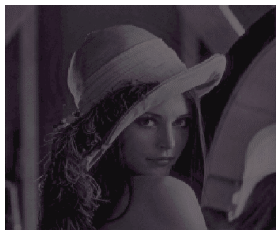

8)

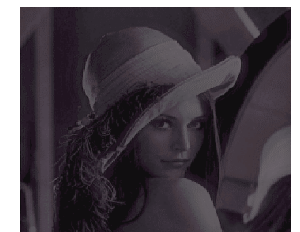

9)

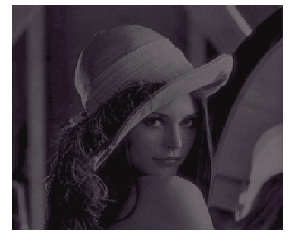

10)

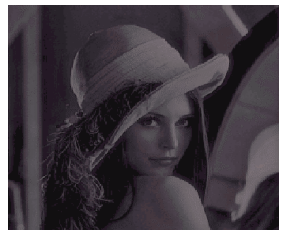



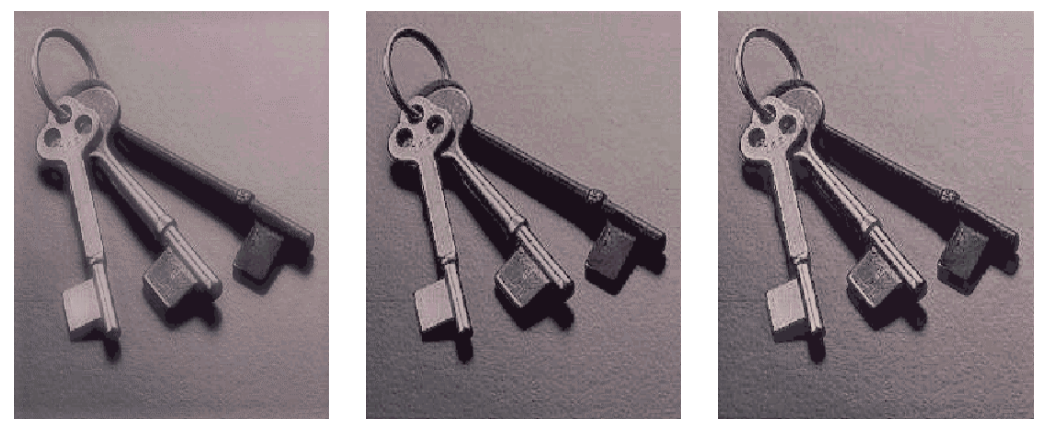